\title{
Thyroid carcinoma and its treatment
}

\author{
SELWYN TAYLOR \\ D.M., M.Ch., F.R.C.S. \\ Dean, Royal Postgraduate Medical School \\ Surgeon, Hammersmith Hospital
}

\section{Summary}

Thyroid cancer is a rare disease. Aetiological factors are increased secretion of TSH and exposure of the growing gland to ionizing radiation. Pathological classification is in two main groups: differentiated and undifferentiated. Differentiated is subdivided into papillary, follicular and medullary.

Papillary and medullary carcinomas occur in young patients, grow slowly, spread by lymphatics. Treatment is surgical excision of thyroid and affected nodes plus thyroid hormone replacement. Follicular carcinoma spreads by blood to lungs and bones. Treatment is total thyroidectomy and radioiodine therapy of the metastases followed by thyroid hormone replacement.

Undifferentiated tumours occur in old patients. They are identified by drill biopsy and treated with radiotherapy.

\section{Introduction}

It is remarkable that a disease which is only responsible for approximately 300 deaths a year in England and Wales with a population of nearly 50 million should command so much attention, but since I last wrote for the Postgraduate Medical Journal on the self-same subject in 1951 (Taylor, 1951) many new and interesting observations have been reported. It is now apparent that increased secretion of thyroid stimulating hormone, TSH, and exposure to ionizing radiation in infancy are important aetiological factors. The classification of Woolner and his colleagues at the Mayo Clinic has now received almost universal recognition. A new clinicopathological variety has been identified, the medullary carcinoma, with some unusual features. Of especial importance to the clinician, the indications for using the four main therapeutic tools, surgery, radioactive iodine, thyroid hormone and radiotherapy have become much clearer. This article is prepared in the light of a recent survey of a personal series of 222 patients with malignant tumours of the thyroid seen over the period 1950-66 (Taylor \& Davis, 1968).

\section{Classification}

In 1931 Dunhill classified thyroid carcinomas into two main groups, differentiated and undif- 0 ferentiated. In general terms, differentiated thyroid carcinoma is a disease of younger people, spreads slowly and usually carries a good prognosis. Undifferentiated carcinoma is seen in the over-60-year-olds, grows swiftly and almost always kills the patient. Useful additions to this classification have been concerned with subdividing the differentiated group and the one which now commands almost universal accepte ance is that of Woolner and his colleagues a the Mayo Clinic (Woolner et al., 1961):

Differentiated

Papillary

Follicular

Medullary

Undifferentiated

In a masterly study based on 885 cases observed over a 30-year period they pointed out that these tumours which showed papillary elements behaved as if the whole tumour was a papillary one, even though the majority of the tissue was follicular in pattern.

All thyroid diseases show a preponderance of female to male cases and in our own clinic the ratio both for simple and toxic goitre is approximately $8: 1$. However, with thyroid carcinoma there were 162 females and sixty males, a ratio of less than $3: 1$. In other words the sex incidence of carcinoma seems to be much less heavily inclined to the female than occurs with other thyroid conditions. There is an interesting point here which again points up the difference between the differentiated and undifferentiated tumours: the sex ratio for the undifferentiated tumours was almost 5:1 while for the differentiated it was little more than $2: 1$, femalemale. Some 10 years ago it was pointed out from this clinic that the different types of thy- 
roid carcinoma tend to occur in characteristic age groups (Alhadeff, Scott \& Taylor, 1956). On the whole, papillary carcinoma typically occurs in the younger patients and all the thyroid carcinomas seen in children were of this type. The follicular carcinomas were seen in an older age group while lastly the undifferentiated tumours occurred most frequently after the age of 60 . Medullary carcinoma is such a rare condition and is compatible with such long survival, that it would be unwise to allot it a place in this timescale, although my guess would be that it is similar to the papillary group.

\section{Aetiology}

The control of thyroid secretion is mediated through a feed-back mechanism between the thyroid stimulating hormone, TSH, of the anterior pituitary, and thyroxine and triiodothyronine production by the thyroid gland. Thus a rise or fall in the normal level of circulating thyroid hormone leads to a fall or rise in TSH secretion. In the great endemic goitre areas of the world: the Andes of South America, Himalayas in Northern India, New Guinea Highlands and Uele region of the Congo, iodine deficiency leads to intense TSH stimulation and the entire population have hyperplastic thyroid glands which are usually enlarged. It might well be expected that the incidence of neoplasia would be higher in these circumstances, but comparative statistical studies have given conflicting results. Pendergrast, in an exhaustive review of United States figures (Pendergrast, Milmore \& Marcus, 1961), could find no direct relationship, nor could the World Health Organization study group on endemic goitre, which resulted in WHO monograph 44 (Endemic Goitre, 1960), deduce with certainty that thyroid cancer was commoner in endemic areas. However, as long ago as 1928 Wegelin pointed out that the frequency of thyroid cancer found at routine autopsies varied from $1.04 \%$ in Berne, an endemic goitre area, to $0.09 \%$ in Berlin which is non-goitrous. If we take the number of deaths from thyroid cancer for England and Wales in 1956, population $44,300,000$ and compare them with Switzerland, population $4,300,000$ they are practically the same. In other words, Switzerland with its endemic goitre had ten times as many deaths from cancer of the thyroid (Taylor, 1956). Critics point out that criteria of malignancy may not be the same in different countries and even that different types of tumour may occur in endemic areas (Walthard, 1961). In addition, few authors have distinguished between the differentiated and undifferentiated tumours, and it is surely only the differentiated and in particular the follicular carcinomas that one might expect to see more commonly in areas of endemic goitre, where TSH stimulation is higher than normal.

A particularly careful study was published in 1966 from Cali in Colombia (Wahner et al., 1966), a severely goitrous area of the Andes. Compared with data from a non-goitrous area, autopsy records showed a significantly increased frequency and death-rate from thyroid carcinoma and in the relative proportion of follicular carcinomas. Eighty per cent were found in nodular parenchymatous goitres and none in colloid goitres; only papillary carcinoma was found in colloid goitre.

In contrast to the somewhat conflicting evidence presented above, when we turn to ionizing radiation there is no doubt that exposure of the foetal and growing thyroid to even small doses, can lead to carcinoma (Taylor 1965a). It was in 1950 that Duffy \& Fitzgerald first suggested this relationship as a result of the development of thyroid cancer in children following thymic irradiation in infancy. Clark in 1955 described a further fifteen cases of thyroid cancer in the young, all of which had been associated with $\mathrm{X}$-irradiation to the chest during the first years of life. In 1957, Simpson \& Hempelmann reported a controlled series of over a thousand children who had received X-ray treatment for so-called enlarged thymus in early childhood with a similar number of their siblings who had not. This conclusively showed a higher number of carcinomas in those who had received the irradiation. Experimental confirmation was convincingly presented by Doniach who gave rats thiouracil in their drinking water to stimulate hyperplasia and growth of the thyroid and then exposed the gland to small doses of $X$-rays or radioactive iodine in the weanling period (Doniach, 1950). The irradiated rats produced more tumours and a number of cancers. Recently the author (Taylor, 1965b) showed that rats irradiated at the weanling stage and subsequently fed on a low iodine diet developed thyroid nodules, some of which were frankly malignant, in a much higher proportion than those which did not receive irradiation.

The third aetiological factor in thyroid carcinoma is the genetic one. Hyperthyroidism and simple goitre are both known to occur with great frequency among the members of certain families and in the case of Hashimoto's disease, a high prevalence of autoantibodies has been reported among the close relatives. It is not surprising therefore that papillary carcinoma has been found in the thyroid glands of identical 
twins (Robinson \& Orr, 1957) and the author has seen hyperthyroidism and thyroid carcinoma and simple goitre and carcinoma, occurring in close members of the same family on a number of occasions.

\section{Diagnosis}

Thyroid carcinoma should always be suspected when a nodule in the gland feels very hard, especially if that nodule is solitary. In a recent survey of 207 consecutive clinically solitary nodules in the thyroid gland, 124 were found to be solitary at operation and 108 were considered solitary by the pathologist (Taylor \& Psarras, 1967). This means that about $50 \%$ of the nodules that felt solitary were so. Of these 207 nodules twenty-six were malignant, an overall incidence of $12.6 \%$ and all were differentiated carcinomas; fifteen papillary and eleven follicular. The younger the patient the more likely is it that the nodule will be malignant. The reason why solitary nodules at the extremes of life are more likely to be malignant is because of the rarity of benign nodules in these age groups as the accompanying graph (Fig. 1) clearly demonstrates.

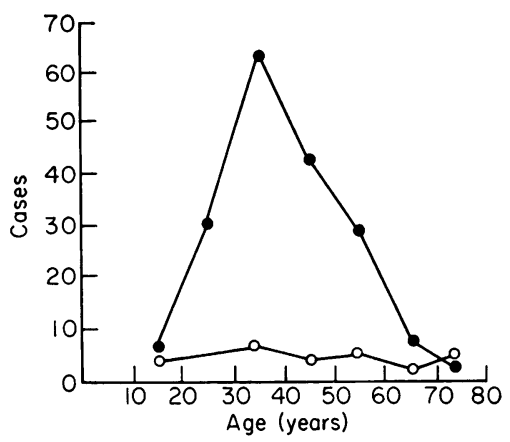

FIG. 1. Age distribution in 207 benign and malignant nodules. Benign (181); O, malignant (26). (From Praxis, 1967, 56, 370, by kind permission.)

Hoarseness accompanying a thyroid enlargement is pathognomonic of malignancy since it betokens involvement of a recurrent laryngeal nerve. Similarly, fixation of the gland to surrounding tissues and the involvement of regional lymph nodes indicates a carcinoma.

A good case-history should always be obtained with special note of where the patient was born and bred, any family history of goitre and of irradiation to neck and chest in childhood. In addition to careful palpation of the neck, a general examination must be made and chest and neck X-rayed. Soft tissue shadows will show the extent of the goitre, reveal calcification which is often seen in papillary and medullary carcinomas and spread to mediastinum and lung fields. It also shows any deviation and compression of the trachea. A tracer dose of radioactive iodine is given and the uptake measured at $48 \mathrm{hr}$ and the neck scanned. Cold areas may be the site of carcinoma, but may equally well be caused by haemorrhage and degeneration, recent or old. Hot areas are never the site of carcinoma. Blood is taken for the protein bound iodine, PBI, which is a useful baseline for replacement thereapy after operation. The serum calcium is determined for the same reason and also thyroid antibodies, since occasionally a florid attack of Hashimoto's thyroiditis mimics carcinoma. All patients should have their vocal cords examined by indirect laryngoscopy before operation in case there is a unilateral palsy already present. When this is found to be so it is imperative that the nerve on the opposite side be preserved.

Since the treatment of thyroid carcinoma depends on the pathological type, each variety will be considered in turn.

\section{Papillary carcinoma}

Papillary carcinoma is a disease of young adults and occasionally children which is typically slow-growing and spreads by the lymphatics? Most papillary tumours show areas of a follicularo pattern and many follicular tumours show papil? lary areas. When they do, they behave in the same way as papillary growths. We have studied the pathways of lymphatic drainage by injecting patent blue dye into the thyroid gland on the operating table (Richardson, 1964). There is a rich intra-glandular network which explains the common finding of multiple foci of carcinoma. Outside the gland, nodes are seen around the inferior thyroid arteries, in the groove between oesophagus and trachea, above the isthmus (Delphian nodes), beside the superior vascular pedicles and in association with the thymus. Submandibular nodes may be involved and later the jugular chains and supraclavicular nodes.

In the past the finding of well-differentiated thyroid carcinoma in the lateral triangles of the neck was considered to be some aberration of embryological development, the so-called lateral aberrant thyroid. Today it is appreciated that such a finding always indicates that there is a primary focus of carcinoma in the thyroid, although this may be only microscopic in size, the occult carcinoma of the thyroid.

Papillary carcinomas may behave in a way quite unlike that of other malignant tumours. Small tumours may remain stationary for many years or may be discovered incidentally at 
autopsy. Similarly, metastatic deposits in cervical lymph nodes may remain for as long as 20 years without further spread. On the other hand, the occasional papillary tumour is aggressive and metastasizes rapidly or may invade the trachea, a condition usually seen in male patients around the age of 20 . It is not surprising, therefore, that widely divergent opinions are held on the treatment of this protean disease.

\section{Treatment}

If at operation a solitary nodule is exposed which raises the suspicion of carcinoma and if to the eye and the exploring finger there is no evidence of spread to other tissues including the nearby lymph nodes, then total lobectomy is done preserving the recurrent nerve and the parathyroids. When the pathologist reports a papillary carcinoma the patient is given full thyroidhormone replacement daily and carefully examined at 3, 6, 9 and eventually 12-monthly intervals for the rest of his or her life. It is among this group that we have obtained the best results in papillary carcinoma. It is unnecessary to have facilities for a skilled opinion on a frozen section and there must be very few centres where it is available for this particular disease. For cosmetic reasons total lobectomy should always be combined with removal of the isthmus and a thin slice from the opposite lobe, since this gives a better looking neck should hypertrophy of the remaining thyroid tissue occur (Taylor, 1965a).

When a papillary carcinoma has spread outside the confines of the thyroid capsule it usually invades nearby lymph nodes which enlarge and feel firm; it may also grow directly into the trachea with which it shares abundant lymphatic channels. If the surgeon is confident that such spread has occurred, or if a frozen section of a lymph node shows metastatic tumour, then total thyroidectomy should be done with preservation of recurrent laryngeal nerves and parathyroids, but removal of all nodes which appear involved. No advantage is gained by embarking on radical neck-dissection since excision of sterno-mastoids and the adjoining tissue-planes will make it no easier to remove the nodes described above and will inevitably lead to scarring and disability in the neck of a young woman or man. The nodes in this disease are enveloped in neat fascial pockets which makes removal easy, unlike the spread of epithelioma from the buccal cavity which demands block dissection.

When the involved nodes are cystic the prognosis is excellent because it means that the carcinoma is well-differentiated with follicles secreting colloid. Under such circumstances the giv- ing of full thyroid-hormone replacement by mouth may be sufficient to inhibit further growth ; in addition the metastases may be differentiated well enough to take up radioactive iodine so that they may be destroyed by this form of irradiation. Details of this form of therapy will be found under follicular carcinomas below, since it is in that form of the disease that the most striking successes are occasionally obtained.

When the wall of the trachea is involved by direct extension it must be excised with the tumour. The resulting defect can then be used as a tracheostomy with prospect of closing later. Alternatively if the involvement is posterior, the defect may be closed and a formal tracheostomy made anteriorly. Rarely, a permanent tracheostomy has to be provided. Tracheal reconstruction can be tried with a prosthesis such as a piece of a Teflon aortic graft, we have had one remain in place for almost 2 years when the disease recurred and needed more extensive excision.

An interesting side-light has been thrown on the metastasis of the thyroid in the neck by Gricouroff of Paris (1966). On carefully examining the lymph nodes removed at block dissection for malignant disease he dicovered in three specimens, tiny groups of thyroid follicles which looked morphologically normal. How did they get there? he likened the condition to endometriosis and named it benign metastatic thyroidosis. It is probably wise not to attach too much significance to this finding until it has been reported more frequently than at present.

External radiotherapy plays very little part in the treatment of papillary carcinoma because it proves so resistant of it. It can prove useful with the less well-differentiated tumours when they spread to sites inaccessible to the surgeon. Papillary carcinoma only spreads by the blood stream late in the course of the disease, but when it does, the speed of growth seems to accelerate.

\section{Follicular carcinoma}

Follicular carcinoma affects an older agegroup than papillary carcinoma and spreads typically by the blood stream to the skeleton and the lungs. The primary tumour is usually well encapsulated and seldom does more than displace the trachea. Spread to the lymph nodes is occasionally seen. Follicular carcinoma can be subdivided into two distinct groups, both on clinical and to some extent on morbid anatomical grounds. In one group, after removal of the primary growth there is no further evidence of the disease. In these patients the primary tumour shows no invasion of the capsule or of blood 
vessels. In the other group, the thyroid capsule and blood vessels show invasion by carcinoma cells and this is often accompanied by pleomorphism of the follicular cells. Mitoses may be seen, a rare finding in the well-differentiated tumour. Such less well differentiated tumours may metastasize rapidly to the skeleton and lungs. There has always been much speculation as to whether these follicular carcinomas arise as a malignant change in a pre-existing benign adenoma. It is hard to see how the problem will ever be solved; my own guess would be that they are malignant from the start, but that their rate of growth and dissemination is influenced by factors of which we at present know little.

About $50 \%$ of patients with follicular carcinoma will present in the first place because of symptoms or signs produced by metastases. The bones most often affected are the flat bones, vertebrae and femora. Backache due to collapse of a vertebra, pain in the ribs and pelvic girdle due to bone destruction and pathological fracture of the femur are the commonest, closely followed by bony swellings of the skull and sternum. Multiple deposits in the lungs can produce haemoptysis, dyspnoea and occasionally a widespread machinery murmur due to the vascularity of the secondaries. Radiologically they may be mistaken for sarcoidosis or tuberculosis.

\section{Treatment}

The treatment of follicular carcinoma of the thyroid is total thyroidectomy in order to remove both the primary lesion which may metastasize and also all normal thyroid tissue which would otherwise compete for the therapeutic doses of radioactive iodine if it should prove desirable to treat the metastases in this way.

Following total thyroidectomy and in the absence of any detectable secondaries, full doses of thyroid hormone are given daily for the rest of the patient's life and a regular clinical examination is done exactly as with papillary carcinoma. It is advisable to have the chest $\mathrm{X}$ rayed each year and if there is any complaint of pain in the bones that area should also be X-rayed.

If metastases are present the patient is not given thyroid replacement after total thyroidectomy but is either allowed to pass into a mildly hypothyroid state which takes 2-3 months, or preferably is given two daily injections of thyrotropin TSH, intramuscularly of 10 i.u. (international units) followed by a large tracer dose of $\frac{1}{2}$ or $1 \mathrm{mCi}$ of ${ }^{131}$. Forty-eight hours later using Pochin's technique the patient is laid on a special table beneath which a collimated count- ing device which measures a thin wedge of radiation right across the patient, is mechanically driven from head to foot (Pochin, 1959). The resulting pattern of uptake is recorded in graph form, the so-called profile scan, and indicates very neatly if there is significant uptake in any of the areas of metastasis. If more than 10-15\% of the dose remains in the patient at 48 hours there is a good prospect of treating the secondaries with radioactive iodine. A maximum of $200 \mathrm{mCi}$ of ${ }^{131} \mathrm{I}$ is given by mouth, but in any case not more than will produce $400-500$ rads of whole body radiation. With such levels of dosage it is best to admit the patient to a special unit since the urine will have to be stored for about 10 half-lives ( 80 days) until it is safe to discharge it into the main drainage. Heavily contaminated clothing or bedding has also to be stored. There is, however, no hazard to doctors and nurses who have to come in close contact with such patients. The treatment is repeated at 6 months and yearly so long as there is uptake of $10 \%$ or more of the dose, or evidence of good uptake on scanning over the lesion. Pochin goes on giving therapeutic doses of the isotope long after the uptake has fallen below these levels and continues with $10 \mathrm{mCi}$ test doses 1 , 2,3 and 5 years thereafter if the patient survives (Pochin, 1967). Agreement has not been reached as to what constitutes the ideal isotope? therapy at present (Smithers, Howard \& Trott, 1965).

Once the metastases have taken up the radioactive iodine, the patient is given full thyroidhormone replacement and this is continued always, except when it is desired to test for uptake of isotope again. When this type of treatment is successful it is among the most dramatic known ; we have had a patient with paraplegia who completely recovered and was well for some years. Unfortunately the majority of such patients, whether their tumours are papillary or follicular, do not take up the isotope. Radiotherapy is then the main line of treatment for the metastases: it usually alleviates the pain but seldom controls the disease.

There is one small group of patients, usually children, with multiple secondary deposits in the lungs in whom, as first shown by Dunhill, giving thyroid hormone replacement by mouth is adequate to shrink the secondaries and prevent further growth of them (Dunhill, 1937). We have only treated one child in this category and as radioactive iodine had already been used elsewhere, it is probable that the resulting fibrosis prevented a very good response, but the improvement was dramatic. 
There are certain hazards with radioiodine therapy for thyroid carcinoma. Total dosage must be such that whole-body irradiation does not exceed 400 rads. If there are any secondaries in the lung fields the dosage must be such that the fibrosis resulting from the irradiation does not reduce the ventilating capacity below that which will sustain life (Rall et al., 1957). Similarly, guarded dosage is called for if there are multiple metastases in the marrow or aplastic anaemia will result. A few patients will develop leukaemia and this hazard must be accepted in the treatment of an otherwise fatal condition.

\section{Medullary carcinoma}

Medullary carcinoma was first described as a clinico-pathological entity by Hazard under the name solid carcinoma with amyloid (Hazard, Hawk \& Crile, 1959) and since then confirmation of this as a clinical entity has come from all over the world. Williams has reported a series of more than eighty patients with this condition (Williams, Brown \& Doniach, 1966). It affects young patients but is so slow-growing that it may be encountered at any age. It spreads by lymphatics with a predilection for the mediastinum; it is only late in the disease that widespread metastasis occurs. It is often heavily calcified and therefore shows up on radiography. Under the microscope it looks very malignant, but its slow growth-rate, sometimes over a period of 20 or 30 years, belies this. An interesting but distinct variant occurs in certain families, the carcinoma being associated with multiple neuromata of the eyelids, tongue and other mucous membranes and also with the development of phaechromocytomas in the adrenal glands. Some of these patients have intractable diarrhoea which is relieved when the tumour and the bulk of the metastases are excised (Williams, 1966). The diagnosis of medullary carcinoma should be suspected when the history is long, the thyroid mass stony hard and extending into the mediastinum and is calcified. Hypertension or a high urinary vanillyl mandelic acid, VMA and diarrhoea are other clues.

\section{Treatment}

The treatment of medullary carcinoma is above all things surgical. Complete removal of the mass in the neck and mediastinum appears to offer a real prospect of cure and even subtotal removal is worthwhile and can offer longterm palliation. In our clinic radiotherapy has had no effect that is detectable and none of these tumours has ever been shown to take up radio-iodine.

\section{Undifferentiated carcinoma}

Undifferentiated or anaplastic carcinoma of the thyroid is one of the most aggressive of man's malignant tumours and typically occurs in the over 60-age-group, being much commoner in females. The usual story is that an older patient complains of rapid enlargement of a goitre with pain which radiates to the ears and hoarseness. Diagnosis is best confirmed by drill biopsy (Deeley \& Pollock, 1966) followed by radiotherapy of 4000-5000 rads delivered through a variety of fields to the neck and upper mediastinum. These tumours never take up radio-iodine and all manner of surgical operations, no matter how radical, do nothing to alleviate the patient's misery, indeed they may make the condition less tolerable. The immediate response to irradiation may be good but the growth soon becomes resistant and the major complication is almost always obstruction to the trachea. Sooner or later a tracheostomy has to be done, but on the whole it is best to defer this as long as breathing is not too difficult.

If survival from anaplastic carcinoma extends longer than 1 year it is as well to review the original histological sections. In our own series this has almost always resulted in a change of diagnosis, usually to medullary carcinoma. Rarely a florid example of Hashimoto's thyroiditis can mimic carcinoma but the differential diagnosis should not long be in doubt.

\section{Thyroid hormone replacement}

More than 10 years ago we gave up using dried thyroid extract because of the lack of uniformity in potency and employed the synthetic sodium-1thyroxine which costs very little more. In 1959 we started using a mixture of thyroxin and triiodothyronine (Taylor, 1961) since this more closely resembled what circulates normally in the body and the patients are less likely to give up taking the pills because they miss immediately the energizing effect of the $T_{3}$. More recently we have raised the amount of $T_{3}$ in the mixture with the result that if the patient takes sufficient replacement to become euthyroid then the level of PBI will be within the normal range. If thyroxine alone is used, levels of $10-12 \mu \mathrm{g}$ PBI result and with pure $T_{3}$ the PBI can be as low as 2 or even $1 \mu \mathrm{g}$. The mixture is prepared by Glaxo under the name Diotroxin: the usual dose after total thyroidectomy is $0.3 \mathrm{mg} /$ day. A very large patient may require as much as $0.4 \mathrm{mg} /$ day and most people need to reduce the dose in the tropics or extremely hot weather. 


\section{References}

AlhadefF, R., ScotT, F. \& TAYlor, S. (1956) A clinicopathological study of thyroid carcinoma. Brit. J. Surg. 43, 617.

Clark, D.E. (1955) Association of irradiation with cancer of the thyroid in children and adolescents. J. Amer. med. Ass. 159, 1007.

Deeley, T.J. \& Pollock, D.J. (1966) Experiences with a high speed pneumatic drill biopsy machine. Brit. J. Cancer, $20,442$.

DoNIACH, I. (1950) The effect of radioactive iodine alone and in combination with methylthiouracil and acetylaminoflourine upon tumour production in the rat's thyroid gland. Brit. J. Cancer, 4, 223.

Duffy, B.J., JR and Fitzgerald, P.J. (1950) Cancer of the thyroid in children, a report of 28 cases. J. clin. Endocr. 10, 1296.

Dunhill, T.P. (1931) Carcinoma of the thyroid gland. Brit. J. Surg. 19, 83.

DuNHILL, T.P. (1937) The surgery of the thyroid gland. Lettsomian lecture. Trans. med. Soc., Lond. 60, 234.

Endemic Goitre (1960) Monograph 44, World Health Organization, Geneva.

GricouROFF, G. (1966) The problem of lateral aberrant thyroids, its interpretations Int. Coll. Tumour Thyroid Gland, Marseilles, p. 29. Karger, Basel.

Hazard, J.B., HaWK, W.A. \& CRILE, G., JR (1959) Medullary (solid) carcinoma of the thyroid-a cliniopathologic entity. J. clin. Endocr. 19, 152.

Pendergrast, W.J., Milmore, B.K. \& Marcus, S.C. (1961) Goiter, thyrotoxicosis and cancer. J. chron. Dis. 13, 22.

Pochin, E.E. (1959) Profile counting in Medical Radioisotope Scanning, p. 143. International Atomic Energy Authority, Vienna.

PochIN, E.E. (1967) Prospects from the treatment of thyroid carcinoma with radioiodine. Clin. Radiol. 18, 113.

Rall, J.E., Alpers, J.B., Lewallen, C.G., Sonenberg, M., Berman, M. \& RAWSON, R.W. (1957) Radiation pneumonitis and fibrosis: a complication of radio-iodine treatment of pulmonary metastases from cancer of thyroid. J. clin. Endocr. 17, 1263.

RicharDson, P.J. (1964) A study of the lymphatic drainage of the human thyroid. Brit.J. Surg. 51, 73.
Simpson, C.L. \& HempelmanN, L.H. (1957) The association of tumours and roentgen ray treatment of the thorax in infancy. Cancer, 10, 42.

Smithers, D.W., Howard, N. \& TrotT, N.G. (1965) Treatment of carcinoma of the thyroid with radioiodine. Brit. med. J. ii, 969.

TAYlor, S. (1951) Carcinoma of the thyroid. Postgrad. med. J. $27,54$.

TAYLOR, S. (1956) Physiologic considerations in the genesis and management of nodular goiter. Amer. J. med. 20, 698.

TAYLOR, S. (1961) A new thyroid preparation. Lancet, i, 341.

TAYLOR, S. (1965a) Surgical treatment of carcinoma of the thyroid. Brit. J. Surg. 52, 740.

TAYLOR, S. (1965b) Induction of thyroid cancer in rats on low-iodine diet. Current Topics in Thyroid Research (Ed. by C. Cassano and M. Andreoli). Academic Press, Rome.

TAYloR, S. \& DAvis, P.W. (1968) A review of the treatment of 222 patients with malignant tumours of the thyroid. In: Tumours of the Thyroid Gland (Ed. by D. Smithers). Chapter 19. Butterworths, London.

TAYlor, S. \& Psarras, A. (1967) The solitary thyroid nodule -benign or malignant. Praxis, 56, 370.

Wahner, H.W., Cuello, C., Correa, P. Uribe, L.F. \& GaITAN, E. (1966) Thyroid carcinoma in an endemic goiter area, Cali, Colombia. Amer. J. Med. 40, 58.

WALTHARD, B. (1961) The influence of iodine prophylaxis of goitre on the frequency of cancer of the thyroid gland and on its structure Advances in Thyroid Research (Ed. by R. Pitt-Rivers). Pergamon Press, London.

Wegelin, C. (1928) Malignant disease of the thyroid gland and its relations to goitre in man and animals. Cancer Rev. 3, 297.

Williams, E.D. (1966) Diarrhoea and thyroid carcinoma Proc. R. Soc. Med. 59, 602.

Williams, E.D., Brown, C.L. \& Doniach, I. (1966) Pathological and clinical findings in a series of 67 cases of medullary carcinoma of the thyroid. J. clin. Path. 19, 103.

WOOLNER, L.B., Beahrs, O.H., BlaCK, B.M., McConahey, W.M. \& KeATING, F.R. (1961) Classification and prognosis of thyroid carcinoma. A study of 885 cases observed in a thirty year period. Amer. J. Surg. $102,354$.

Robinson, D.W. \& ORR, T.G. (1957) Carcinoma of the thyroid and other diseases of the thyroid in indentical twins. Arch. Surg. 70, 923. 